Neonatology-then and now (CHM Walker)

Neonatal sepsis (1960)

\title{
Two outbreaks of neonatal skin sepsis caused by Staphylococcus aureus, phage type 71
}

\author{
C $\mathrm{H}$ L HOWELLS AND H EVERLEY JONES
}

\author{
The Royal Hospital, Wolverhampton (Arch Dis Child 1961;36:214-6)
}

One aspect of neonatal care which has not changed with the passage of time is the ever present threat of infection. The subject of this paper was a constant problem in nurseries at the time and paediatricians were learning of the ubiquitous nature of Staphylococcus aureus and Candida albicans. Staphylococcal pneumonia, meningitis, and osteomyelitis were not uncommon and the skin as an avenue of infection was always a worry.

In this paper 14 cases of major bullous lesions or abscesses caused by $S$ aureus occurred in one hospital over a period of 15 days. $S$ aureus was isolated also from one ward sister, a window ledge, and a partition. In the other hospital two cases occurred on the same day; a mother and a ward sister were found to be carriers and the ward was immediately closed. Efforts were directed towards the prevention and control of outbreaks of this kind and the management advice offered here was as follows:

'The organism isolated was found to be penicillin sensitive in all except one infant. Penicillin was therefore given to all except the most mildly affected. The skin lesions were treated with local applications of flavine or gentian violet in spirit. The latter seemed to be rather more effective. The carriers were treated with graneodin applied to the anterior nares, and excluded from duty until their nasal swabs were clear. When the second cases occurred, the practitioners attending infants discharged subsequently were asked to notify the hospital if any infection developed in either mothers or their infants. Only one such came to light, a single spot which rapidly disappeared. The wards concerned were closed to further admissions and the walls thoroughly cleaned, using 1:30 'savlon'. Following this, redecoration was undertaken.

The blankets and mattresses used were stoved. Subsequently, cotton cellular blankets were introduced. The use of hand creams and soaps containing chlorohexidine was encouraged.
It is our impression that, following these outbreaks, there is greater awareness of the possible dangers amongst the nursing staff, and there have been no further cases.'

Today. Where good general management and hygiene exists in the postnatal ward serious skin infections in the term baby have been reduced to a minimum and outbreaks such as those described in this paper are rare. But how things have changed in the special care baby units and intensive care units! Gone are the caps, masks, and overboots-and white coats or gowns seem to be a matter of personal choice. The nursery doors are open to all and sundry (almost) and checking staff for carrier state of organisms dangerous to the baby has been abandoned. This has made life very much easier for those working in and visiting these units, but it has also made it more difficult for everyone to remain vigilant and ensure scrupulous hand washing and exclusion of the obviously infected adult or sibling.

The type of organism may have changed but outbreaks of infection in these units still occur. Many are as, or even more, life threatening and difficult to eradicate than the old staphylococcus. The organisms are often less sensitive to available antibiotics and viruses lurk in the background waiting their chance to produce pneumonia and myocarditis. While attempts are being made to reduce some of these problems by reducing the duration of courses of antibiotics, ${ }^{1}$ and while the value of routine immunoglobulin remains uncertain, ${ }^{2} 3$ the battle between wily organisms and pharmaceutical scientists loses none of its intensity.

\footnotetext{
References

1 Isaacs D, Wilkinson AR, Moxon ER. Duration of antibiotic courses for neonates. Arch Dis Child 1987;62:727-8.

2 Conway SP, Gillies DRN, Docherty A. Neonatal infection in premature infants and use of human immunoglobulin, Arch Dis Child 1987;62:1252-6.

${ }^{3}$ Stabile A, Sopo SM, Romanelli V, Pastore M, Pesaresi MA. Intravenous immunoglobulin for prophylaxis of neonatal sepsis in premature infants. Arch Dis Child 1988;63:441-3.
} 